\title{
Bootstrap determination of the reliability of $b$-values: an assessment of statistical estimators with synthetic magnitude series
}

\author{
Mendy Bengoubou-Valérius • Dominique Gibert
}

Received: 7 September 2011/Accepted: 28 August 2012

(C) Springer Science+Business Media B.V. 2012

\begin{abstract}
We consider some practical issues of the determination of the $b$-value of sequences of magnitudes with the bootstrap method for short series of length $L$ and various quantization levels $\Delta m$ of the magnitude. Preliminary Monte Carlo tests performed with $\Delta m=0$ demonstrate the superiority of the maximum likelihood estimator $b_{\mathrm{MLE}}$, and the inconsistency of the, yet often used, $b_{L R}$ estimator defined as the least-squares slope of the experimental Gutenberg-Richter curve. The Monte Carlo tests are also applied to an estimator, $b_{\mathrm{KS}}$, which minimizes the Kolmogorov-Smirnov distance between the cumulative distribution of magnitudes and a power-law model. Monte Carlo tests of discrete versions of the $b_{\mathrm{MLE}}$ and $b_{\mathrm{KS}}$ estimators are done for $\Delta m=\{0.1,0.2,0.3\}$ and used as reference to evaluate the performance of the bootstrap determination of $b$. We show that all estimators provide $b$ estimates within $10 \%$ error for $L \geq 100$ and if a large number, $n=2 \times 10^{5}$, of bootstrapped sample series is used. A resolution test done with $\Delta m=0.1$ reveals that a clear distinction between $b=0.8,1.0$, and 1.2 is obtained if $L \geq 200$.
\end{abstract}

Keywords Power law $\cdot$ Bootstrap $\cdot b$-values $\cdot$ Earthquake series

\section{Introduction}

The space and time variations of the seismic parameters in a given region are often considered as indicators of changes in the seismicity regime, eventually announcing the occurrence of a large earthquake (e.g. Schorlemmer et al. 2010). Among the seismic parameters whose variations are studied, the most popular are certainly the amplitude,

\footnotetext{
M. Bengoubou-Valérius

Bureau de Recherche Géologiques et Minières Guadeloupe, Morne Houëlmont, Route de l'Observatoire, 97113 Gourbeyre, France

e-mail: m.bengoubou-valerius@brgm.fr

D. Gibert $(\bowtie)$

Institut de Physique du Globe de Paris (CNRS UMR 7154), Sorbonne Paris Cité, 1 rue Jussieu, 75238

Paris cedex, France

e-mail: gibert@ipgp.fr
} 
$A$, and the exponent, $b$, of the Gutenberg-Richter (GR) power-law distribution of earthquake magnitudes (Gutenberg and Richter 1944; Main 2000),

$$
N(m \geq M)=A \times 10^{-b M},
$$

where $N(m \geq M)$ is the number of earthquakes with a magnitude $m$ greater than or equal to $M$. The amplitude $A$ fixes the rate of seismicity while the exponent $b$-also called the $b$-value-controls the proportion of earthquakes with different magnitudes.

Although a value of $b \approx 1$ is observed for the global worldwide seismicity (e.g. Kagan 1999), numerous studies report that the $b$-value may significantly change both in space and in time (e.g. Wiemer and Benoit 1996; Ayele and Kulhánek 1997; Wiemer et al. 1998; Gerstenberger et al. 2001; Schorlemmer et al. 2003). Spatial variations are tentatively considered to depend on the fault heterogeneity (e.g. Meyer et al. 2004), and on the level of stress and pore pressure in the crust (e.g. Scholz 1968; Wyss 1973; Amitrano et al. 2003; Schorlemmer et al. 2005). Temporal decreases in the $b$-value have also been reported before the occurrence of main earthquakes (e.g. Wyss and Habermann 1988; Nuannin et al. 2005). Other studies present small-scale spatiotemporal mappings of the $b$-value under active regions such as volcanoes in order to identify regions of magma migration (Wiemer and McNutt 1997; Wyss et al. 1997, 2001; Murru et al. 2004; McNutt 2005).

The number of studies devoted to time and space variations of the $b$-value regularly increases (e.g. Zöller et al. 2002; Zhao and Wu 2008; Papadopoulos and Baskoutas 2009; Telesca et al. 2009) while a debate remains on the reality and on the physical significance of these variations (e.g. Frohlich 2004). Beside the necessity to progress on the physical understanding of the $b$-value variations, the reliability of these reported variations gains an increasing importance. Indeed, many studies recently published use different $b$ estimators and apply them on eventually short earthquake series (e.g. Bouhadad and Laouami 2002; comment by Peléez Montilla and Hamdache 2004). Because of the power-law behavior of the analyzed series of magnitudes, the statistical determination of $b$ poses specific issues early recognized by Aki (1965) and Utsu (1965) (see also Bender 1983), and more recently by Goldstein et al. (2004) in the general framework of the determination of power-law slopes encountered in many complex systems, physical or not. A recent review of the most popular $b$ estimators, written by Marzocchi and Sandri (2003), presents and discusses the different statistical estimators of $b$ proposed in the seismological literature. In particular, these authors point out that the problem of the experimental determination of the $b$-value remains open (see for instance the case study in the paper by Papadopoulos et al. (2006)) and that no unique practice is in usage in the seismological community, making difficult to compare results from independent studies. Further complications arise in studies of regions with a low seismicity rate (i.e. small $A$ in Eq. 1) where the number of earthquakes is small. Such a situation is not uncommon, even in highly active tectonic areas where low seismicity rate may be observed either in gap regions or in sub-regions to study the seismic parameters of particular faults (e.g. Zhao and Wu 2008).

In the present paper, we aim at contributing to the assessment of a practical methodology for determining $b$-values from short series of quantized magnitudes. Instead of assuming a particular model to derive the statistical characteristics of the $b$ estimators, we use a nonparametric approach, namely the bootstrap method, to directly compute the probability density distributions of $b$-values from a single series of magnitudes. Let us recall that bootstrap consists in producing an ensemble of $n$ magnitude sequences generated by randomly sampling with replacement the single original data series. A key issue of this approach is to verify that the bootstrap resampling procedure correctly reproduces the 
statistical variability of the $b$-values derived from short quantized series. While many examples exist which demonstrate that bootstrap often leads to sound statistical conclusions, the obvious dependence of the obtained results on the original data series implies that we have no guaranty of systematic success. This makes the bootstrap a problemdependent method, both from the view point of the data available and from the view point of the parameters to be derived (Young 1994). In the present paper, we proceed by comparing the results of bootstrapping with Monte Carlo simulations to document the domain where bootstrap may be efficient to recover the statistical characteristics of $b$-value estimators.

The paper is organized as follows: we introduce several classically used $b$-value estimators and performed Monte Carlo checks of their reliability, both in the continuous and in the discrete cases. These tests, performed for series of magnitude of length $L=50,100$, 200 , and 400 , produce probability distributions of the $b$ estimators for both truncated and quantized series of magnitudes. These probability distributions are further used as references which are compared with probability distributions obtained with the bootstrap. In the last section of the paper, we illustrate the resolution power of the method by applying it to synthetic series with different $b$-values. The conclusion summarizes several practical recommendations.

\section{$2 b$-value estimators}

We now introduce the most two popular estimators of the $b$-value, namely the linear regression and the maximum likelihood estimators. In addition, we propose a third estimator based on the Kolmogorov-Smirnov test. In a first step, we present the continuous version of these estimators.

\subsection{Least-squares linear regression estimator (LR)}

The linear regression estimator of $b$ is straightforwardly obtained by rewriting Eq. (1) in the $\log -\log$ domain to obtain a linear relation between $N$ and $M$,

$$
\log [N(m \geq M)]=\log A-b \times M .
$$

From this equation, fitting a straight line to the log-transformed GR law gives the linear regression estimate of $b$ (e.g. Okal and Kirby 1995; Triep and Sykes 1997; Main 2000; Zöller et al. 2002),

$$
b_{L R}=\frac{L \sum_{i=1}^{L} m_{i} \log _{10}\left(N_{i}\right)-\sum_{i=1}^{L} m_{i} \sum_{i=1}^{L} \log _{10}\left(N_{i}\right)}{L \sum_{i=1}^{L} m_{i}^{2}-\left(\sum_{i=1}^{L} m_{i}\right)^{2}},
$$

where $L$ is the number of magnitudes, $m_{i}$, used in Eq. (2). Equation (3) corresponds to the standard least-squares regression case discussed in details by Castellaro et al. (2006) who also give the expressions for the standard deviation associated with $b_{L R}$. Although appealing for its simplicity and easy implementation through line fitting software, many authors point out the pitfalls of the $b_{L R}$ estimator and we refer the readers to the recent study of Goldstein et al. (2004). 


\subsection{Maximum likelihood estimator (MLE)}

The maximum likelihood estimator, $b_{\text {MLE }}$, initially proposed by Aki (1965) and Utsu (1965) is directly derived from the power-law probability density distribution of the magnitudes $m$,

$$
p(m)=b \ln (10) 10^{-b\left(m-m_{z}\right)},
$$

where $m_{z}$ is the threshold magnitude beyond which the recurrence curve is straight. The probability, $l(b \mid \mathcal{M})$, that a given series of magnitudes, $\mathcal{M}=\left\{m_{i}, i=1, \ldots, L\right\}$ is a realization of the stochastic process (4) reads,

$$
l(b \mid \mathcal{M})=\prod_{i=1}^{L} p\left(m_{i}\right)
$$

Inserting (4) in Eq. (5), and taking the $\log$-likelihood $\mathcal{L} \equiv \ln (l)$ gives,

$$
\mathcal{L}(b \mid \mathcal{M})=L \ln b+L \ln [\ln (10)]-b \ln (10) \sum_{i=1}^{L} \ln \left(m_{i}-m_{z}\right) .
$$

The MLE of the $b$-value, $b_{\mathrm{MLE}}$, is such that the log-likelihood is maximum:

$$
\left.\frac{\partial}{\partial b} \mathcal{L}(b \mid \mathcal{M})\right|_{b_{\mathrm{MLE}}}=0
$$

This gives:

$$
\begin{aligned}
b_{\mathrm{MLE}} & =\frac{1}{\ln (10)\left[L^{-1} \sum_{i=1}^{L}\left(m_{i}\right)-m_{z}\right]} \\
& =\frac{1}{\ln (10)\left(\bar{m}-m_{z}\right)},
\end{aligned}
$$

where $\bar{m}$ is the average magnitude of the $L$ events such that $m_{i} \geq m_{z}$. The standard deviation associated with the $b_{\text {MLE }}$ has a close analytical form given by Aki (1965),

$$
\sigma\left(b_{\mathrm{MLE}}\right)=\frac{b_{\mathrm{MLE}}}{\sqrt{L}} .
$$

It should be noted that the $\ln (10)$ factor in Eq. (8) does not appear in the formula given by Newman (2005). This difference explains the 3.04 power-law slope obtained by this author for the distribution of California earthquakes instead of the $b \simeq 1$ value published in seismological studies. A similar $\ln (10)$ normalizing factor is used by Marzocchi and Sandri (2003) and Sornette and Werner (2005). The $b_{\text {MLE }}$ given by Eq. (8) explicitly depends on the threshold magnitude, $m_{z}$, and a wrong choice of this quantity may result in bias in the $b_{\text {MLE }}$ (Wiemer and Wyss 2000). In any case, $m_{z}$ must never-even slightly-be smaller than the magnitude of completeness. The $b_{\mathrm{MLE}}$ is easily obtained through the simple formulas (8) and (9) and it is recommended for its statistical consistency when applied to long data series (e.g. Goldstein et al. 2004) 


\subsection{Kolmogorov-Smirnov estimator (KS)}

A third estimator for $b$ can be obtained by maximizing the Kolmogorov-Smirnov probability that the data obey a power-law behavior. Although suggested by Goldstein et al. (2004), the application of the Kolmogorov-Smirnov test to power-law fitting remains largely ignored and, in the present paper, we shall consider it for comparison with the popular $b_{\mathrm{MLE}}$. Accordingly, the $b_{\mathrm{KS}}$ estimator is defined as the value of $b$ which minimizes the $D$-statistic of the Kolmogorov-Smirnov goodness-of-fit norm,

$$
b_{\mathrm{KS}} \operatorname{such} \min _{b}\left[\sup \left|N_{i}\left(m_{j}>m_{i}\right)-N_{C}\left(m_{i}, b\right)\right|\right] \text {, }
$$

where $N_{C}\left(m_{i}\right)$ is the theoretical power law to be compared with the experimental cumulative curve $N_{i}$, and the sup function returns the largest value in the list. Contrarily to the $b_{\mathrm{MLE}}$, there is no close form for the variance of $b_{\mathrm{KS}}$.

\section{Monte Carlo assessment of continuous $b$-value estimators for short magnitude series}

We now test the performances of the continuous $b$-value estimators presented in Sect. 2 by applying them to an ensemble of $n$ synthetic magnitude series counting a moderate number, $50 \lesssim L \lesssim 400$, of events. The $n b$-value estimates obtained for each estimator and each length $L$ are subsequently used to compute both the mean $\bar{b}$ and its associated standard deviation $\sigma$.

\subsection{Generation of the synthetic sequences}

The Monte Carlo tests have been performed with synthetic series of magnitudes generated with the general branching stochastic process called the epidemic-type aftershock series (ETAS) initially proposed by Ogata $(1985,1988)$. This model accounts for the Omori law which controls the aftershock sequences following every earthquake (Utsu 1961; Enescu et al. 2006). The ETAS model counts few parameters: the base level $R$ of the rate of production of earthquakes, the efficiency $\alpha$ of an earthquake to produce aftershocks, and controls the decay rate $p \gtrsim 1$ of aftershock productivity. Only aftershocks with magnitude larger than $m_{z}$ are produced, and, contrary to the classical definition of aftershocks, the ETAS model allows aftershocks to have a larger magnitude than their triggering earthquake (e.g. Felzer et al. 2002). The total rate of earthquakes of the model is obtained by summing all aftershock series, $a_{i}(t)$, completed by a Poissonian process, $\mu(t)$, representing the seismic activity induced by the regional tectonic loading.

Figure 1a shows an ETAS magnitude series of $N_{T}=5,000$ events generated with the following values of model parameters: $b=1, m_{z}=1.5, \mu=0.005, R=0.04$, $c=0.01, \alpha=0.4$, and $p=1.2$. Figure $1 \mathrm{~b}$ shows the inter-event times of the series. The Gutenberg-Richter curve of this series is shown on the right part of Fig. 1. We find that the results obtained with ETAS sequences and shown in the remaining of the present paper do not differ from those obtained with sequences of magnitudes drawn from a homogeneous power-law model. 
Table 1 Mean (standard deviation) of the $b$ estimators determined for an ensemble of $n=2 \times 10^{5}$ ETAS series of length $L$

\begin{tabular}{llll}
\hline$L$ & \multicolumn{2}{l}{ Monte Carlo statistics } & \\
\cline { 2 - 4 } & $\bar{b}_{L R}(\sigma)$ & $\bar{b}_{\text {MLE }}(\sigma)$ & $\bar{b}_{\mathrm{KS}}(\sigma)$ \\
Eq. $(3)$ & Eq. $(8)$ & Eq. $(10)$ \\
\hline 50 & $0.95(0.24)$ & $1.02(0.15)$ & $1.02(0.18)$ \\
100 & $0.95(0.21)$ & $1.01(0.11)$ & $1.00(0.13)$ \\
200 & $0.94(0.18)$ & $1.00(0.07)$ & $0.99(0.08)$ \\
400 & $0.94(0.14)$ & $1.00(0.05)$ & $1.00(0.06)$ \\
\hline
\end{tabular}

Numbers in parenthesis are one standard deviation

\subsection{Monte Carlo assessment of $b$ estimators}

The three estimators $b_{L R}, \quad b_{\mathrm{MLE}}$ and $b_{\mathrm{KS}}$ have been applied to ETAS series with a number of events $L=50,100,200$ and 400, and Table (1) gives the mean and standard deviation for each estimator and each series length. We observe that $\sigma$ decreases as $L$ increases for all three estimators, indicating that the larger the number of events the smaller the uncertainty over the determined $b$-value. This is, for instance, the case of $\sigma\left(b_{L R}\right)$ which diminishes by a factor of $\approx 1.8$ from $L=50$ to $L=400$. The experimental values of $\sigma\left(b_{\mathrm{MLE}}\right)$ given in Table (1) are remarkably coherent with the values predicted by Eq. (9).

The results of Table (1) show that the mean values $\bar{b}_{L R}$ are biased by about $5 \%$ toward the lower values of $b$ whatever the number of events $L$. The bias in the determination of power-law slopes has recently been reviewed by Goldstein et al. (2004) who show that the biased $\bar{b}_{L R}$ may advantageously be replaced by the maximum likelihood $\bar{b}_{\text {MLE }}$. An extensive comparison of both types of slope estimates performed by Newman (2005) confirms the generally superior performance of the MLE against the LR one. Indeed, the $\bar{b}_{\text {MLE }}$ 's fall very near the theoretical value, $b=1$ (Table 1 ), and the associated standard deviations, $\sigma\left(b_{\mathrm{MLE}}\right)$, are smaller than the $\sigma\left(b_{L R}\right)$ and in full agreement with Eq. (9) which predicts $\sigma\left(b_{\mathrm{MLE}}\right)=0.14, \quad 0.10, \quad 0.07$, and 0.05 for $L=50,100,200$, and 400 , respectively. The results obtained for $b_{\mathrm{KS}}$ are very similar to those for $b_{\mathrm{MLE}}$ with mean $\bar{b}_{\mathrm{KS}} \mathrm{s}$ not significantly different from the $\bar{b}_{\mathrm{MLE}}$ estimates and also falling very near the theoretical value, $b=1$ (Table 1 ). The standard deviations, $\sigma\left(b_{\mathrm{KS}}\right)$, are slightly larger than the $\sigma\left(b_{\mathrm{MLE}}\right)$. However, the results presented here indicate that $b_{\mathrm{KS}}$ is also a good statistical estimator of the $b$-value, but not as intuitive or simple.

\section{Discrete versions of $b$-value estimators}

In the examples of the preceding sections, the magnitudes were assumed perfectly known with a negligible quantization error $\Delta m=10^{-5}$ corresponding to numerical rounding in the computer code. In practice, such an accurate determination of the magnitude is never obtained, and we now assume that the values of magnitudes are quantized in intervals of width $\Delta m$. Consequently, the $L$ values of magnitudes belong to the finite and discrete set $\mathcal{S}=\left\{m_{n}=m_{\min }+n \Delta m\right.$ for $\left.n=0, \ldots, N\right\}$, where $m_{\text {min }}$ is now the smallest quantized magnitude in the catalogue not to be confused with the magnitude of completeness. This quantization of the magnitudes will produce a bias in the $b_{\mathrm{MLE}}$ if the average magnitude, $\bar{m}$, in Eq. (8) is replaced by the average of the quantized magnitudes (see Appendix for 
details). In the remaining of the paper, we shall no more consider the $b_{L R}$ estimator and we refer the interested readers to the detailed study by Castellaro et al. (2006).

\subsection{Discrete MLE estimators}

A first unbiased estimator may be obtained under the assumption that the number, $N$, of bins is sufficiently large, which in practice is satisfied whenever $N \geq 10$ (Appendix). The so-derived estimator reads (Bender 1983),

$$
b_{B}=\frac{1}{\ln (10) \Delta m} \ln \left(1+\frac{\Delta m}{\overline{m_{b}}-m_{\min }}\right) .
$$

This estimator is identical to the one used by Tinti and Mulargia (1987).

A second simplification occurs if $\Delta m \rightarrow 0$. Solving this equation leads to the unbiased $b$-value estimate given by Utsu (1965),

$$
b_{U}=\frac{1}{\ln (10)\left(\overline{m_{b}}-m_{\min }+\frac{\Delta m}{2}\right)} .
$$

Observe that the expression for $b_{U}$ may also be directly obtained by substituting $m_{\min } \rightarrow$ $m_{\min }-\Delta m / 2$ in the formula for the $b_{\text {MLE }}$ (Eq. 8).

\subsection{Discrete KS estimator}

A discrete version, $b_{\mathrm{KSD}}$, of the $\mathrm{KS}$ estimator is obtained by replacing the continuous probability density $N_{C}$ in Eq. 10 with the discrete probability distribution $P_{n}$ given by Eq. 17 in Appendix.

\subsection{Monte Carlo determination of discrete $b$ estimators}

Monte Carlo runs similar to those presented in Sect.3.2 have been performed to assess for the accuracy of the $b_{B}, b_{U}$, and $b_{\mathrm{KSD}}$ estimators. The results are given in Table 2 for the three quantization levels $\Delta m=0.1,0.2$, and 0.3 . The $b_{B}$ estimator has constant good performances whatever the length $L$ and the quantization level $\Delta m$. This estimator also has an associated standard deviation identical to the one for the continuous $b_{\mathrm{MLE}}$. The $b_{U}$ estimator also displays overall good performances with, however, a slight and progressive degradation as long as the quantization level $\Delta m$ increases. The standard deviation for this estimator remains coherent with the values obtained for $b_{\text {MLE }}$. The discrete version of the Kolmogorov-Smirnov estimator, $b_{\mathrm{KSD}}$, shows the paradoxical behavior to have better performances for the roughest quantization level $\Delta m=0.3$. We have no sound theoretical explanation for this result that we suspect to be due to the conservative nature of the Kolmogorov-Smirnov test combined with the diminution of the number of degrees of freedom in the data set as $\Delta m$ increases. The standard deviation of $b_{\mathrm{KSD}}$ is identical to the one of its continuous version $b_{\mathrm{KS}}$ for all tested $L$ and $\Delta m$. However, only the $b_{\mathrm{KSD}}$ obtained for $L=400$ are correct, and this estimator does not appear reliable for smaller values of $L$.

\section{Bootstrap determination of $b$}

We now touch the main objective of the present paper by addressing the practical determination of the $b$-value from a single series of magnitudes with $L$ events. In such a case, 
Table 2 Mean (standard deviation) of the discrete $b$ estimators determined for an ensemble of $n=2 \times 10^{5}$ Monte Carlo (left half of Table) and bootstrap (right half) ETAS series of length $L$, and for quantization levels, $\Delta m=0.1,0.2$, and 0.3

\begin{tabular}{|c|c|c|c|c|c|c|}
\hline \multirow[t]{2}{*}{$L$} & \multicolumn{3}{|c|}{ Monte Carlo statistics } & \multicolumn{3}{|c|}{ Bootstrap statistics } \\
\hline & $\bar{b}_{B}(\sigma)$ & $\bar{b}_{U}(\sigma)$ & $\bar{b}_{\mathrm{KSD}}(\sigma)$ & $\bar{b}_{B}(\sigma)$ & $\bar{b}_{U}(\sigma)$ & $\bar{b}_{\mathrm{KSD}}(\sigma)$ \\
\hline \multicolumn{7}{|c|}{$\Delta m=0.1$} \\
\hline 50 & $1.01(0.15)$ & $1.00(0.15)$ & $0.79(0.18)$ & $1.09(0.16)$ & $1.09(0.16)$ & $0.72(0.21)$ \\
\hline 100 & $1.01(0.10)$ & $1.00(0.10)$ & $0.90(0.12)$ & $0.93(0.08)$ & $0.92(0.08)$ & $0.71(0.12)$ \\
\hline 200 & $1.00(0.07)$ & $1.00(0.07)$ & $0.95(0.09)$ & $0.94(0.07)$ & $0.94(0.07)$ & $0.86(0.10)$ \\
\hline 400 & $1.00(0.05)$ & $1.00(0.05)$ & $0.98(0.06)$ & $1.01(0.05)$ & $1.00(0.05)$ & $0.99(0.07)$ \\
\hline \multicolumn{7}{|c|}{$\Delta m=0.2$} \\
\hline 50 & $1.03(0.16)$ & $1.00(0.15)$ & $0.91(0.18)$ & $1.05(0.14)$ & $1.03(0.13)$ & $0.80(0.18)$ \\
\hline 100 & $1.01(0.10)$ & $1.00(0.10)$ & $0.95(0.12)$ & $1.00(0.09)$ & $0.99(0.09)$ & $0.91(0.13)$ \\
\hline 200 & $1.00(0.07)$ & $0.99(0.07)$ & $0.98(0.08)$ & $1.05(0.07)$ & $1.03(0.07)$ & $1.00(0.09)$ \\
\hline 400 & $1.00(0.05)$ & $0.98(0.05)$ & $0.99(0.06)$ & $1.04(0.05)$ & $1.02(0.05)$ & $0.98(0.06)$ \\
\hline \multicolumn{7}{|c|}{$\Delta m=0.3$} \\
\hline 50 & $1.02(0.15)$ & $0.98(0.13)$ & $0.94(0.17)$ & $0.92(0.13)$ & $0.87(0.12)$ & $0.76(0.15)$ \\
\hline 100 & $1.01(0.10)$ & $0.97(0.09)$ & $0.96(0.12)$ & $1.06(0.12)$ & $1.01(0.11)$ & $1.02(0.15)$ \\
\hline 200 & $1.00(0.07)$ & $0.96(0.06)$ & $0.98(0.08)$ & $0.95(0.07)$ & $0.92(0.06)$ & $0.92(0.08)$ \\
\hline 400 & $1.00(0.05)$ & $0.96(0.05)$ & $0.99(0.06)$ & $1.07(0.06)$ & $1.02(0.06)$ & $1.07(0.07)$ \\
\hline
\end{tabular}

Numbers in parenthesis are one standard deviation

contrarily to the previous Monte Carlo runs, the mean $\bar{b}$ and its standard deviation are evaluated from an ensemble of series obtained by bootstrapping a single original data series. Assuming that the data series is representative of the whole variability of the underlying stochastic process, an ensemble of re-sampled series may be constructed through a combinatorial arrangement of the original data. In such a bootstrap approach, the original data are considered as if they were the entire unknown population. However, even a moderate number $L$ of data involves a huge number, $L^{L}$, of combinations, the full set of re-sampled series is impossible to be exhaustively visited. In order to tackle this practical difficulty, Efron (1982) and others proposed the so-called Monte Carlo bootstrapping which consists in randomly sampling a subset of $n$ series by randomly drawing (with replacement) data from the initial series. Since each re-sampled series counts the same number $L$ of data as the original one, multiple replications of a given magnitude are possible in the resampling. Estimates of the quantities of interest (e.g. $b$-values) are then derived from the set of re-sampled series and can be used to compute various statistical parameters (mean, standard deviation, etc., see e.g. Davison and Hinkley 1997).

Although bootstrap is a popular and commonly used procedure in many fields of science, it must be kept in mind that there is no guaranty that bootstrapping always correctly reconstructs an ensemble of series with statistical properties identical to those for an ensemble of independent series, see for instance the discussion by Abadie and Imbens (2008). The statistical convergence of bootstrapping is particularly problematic for data exhibiting a power-law behavior because of the huge inequitable probability of appearance between small and large magnitude values. Consequently, the larger the magnitude $m_{n}$, the larger the bias expected to alter the number, $N_{n}$, of data with this magnitude.

In the present section, we discuss an experimental assessment of bootstrapping in the particular case of earthquake series with a particular emphasis for short data series. We 
proceed in two stages: (1) we perform bootstrapping of a single series of magnitudes as in real situations, and (2) we perform a series of bootstraps to have a glance at the variability of the results.

\subsection{Bootstrapping $b_{B}, b_{U}$, and $b_{\mathrm{KSD}}$}

In order to mimic a real situation where a single short data series is available, we now apply the bootstrap to a single synthetic ETAS series of length $L=50,100,200,400$, and for quantization levels $\Delta m=0.1,0.2$ and 0.3 . No particular selection is made to obtain the processes series which were randomly drawn in the ETAS sequence of Fig. 1.

The numerous tests performed in the background of the present study clearly indicate that the number $n$ of bootstrap series to generate is of a critical importance to obtain reliable $b$ estimates. In practice, we observed that a huge number $n$ of series is necessary to derive correct results, and in the present study $n=2 \times 10^{5}$. Although much larger than the $n$ values suggested in the literature (e.g. Efron 1992), computations with such a large number of replicas nowadays take a couple of minutes with a laptop computer. The results are given in Table 2 and show that the standard deviations obtained by bootstrapping are in good agreement with their Monte Carlo counterparts. Most $b$ estimates depart by less than $10 \%$ from the true value (i.e. $b=1$ ); however, several biases may be as large as $30 \%$ for the $b_{\mathrm{KSD}}$ estimator which also suffers from the highest standard deviates.

We recall that the results of Table 2 are obtained for a single series of magnitude values for each $L$, and it is of much importance to further explore the nature of the observed biases observed. Are the biases systematically corrupting a particular $b$ estimator or are they specific biases associated with the particular data series analyzed in the example of Table 2? To document this issue, we performed numerous bootstrap runs with different initial data sequences in order to detect the occurrence of systematic biases. The results obtained for a subset of 10 runs are shown in Fig. 2 for the three estimators $b_{B}, b_{U}$, and $b_{\mathrm{KSD}}$. This Figure shows a decrease in the standard deviation of the bootstrap estimators when the series length $L$ increases with values identical to those obtained with Monte Carlo runs (Table 2). Figure 2 also shows that most bootstrap $b$ estimates have the true $b=1$ in their $95 \%$ confidence interval. It may also be observed that the results obtained for the three $b$ estimators look very similar. For instance, when highly biased estimates occur, they are coherently obtained for all three $b$ estimators as, for instance, for $\Delta m=0.2$ and $L=200$ and 400 . Since the same series is used to compute $b_{B}, \quad b_{U}$ and $b_{\mathrm{KSD}}$ for a given $L$
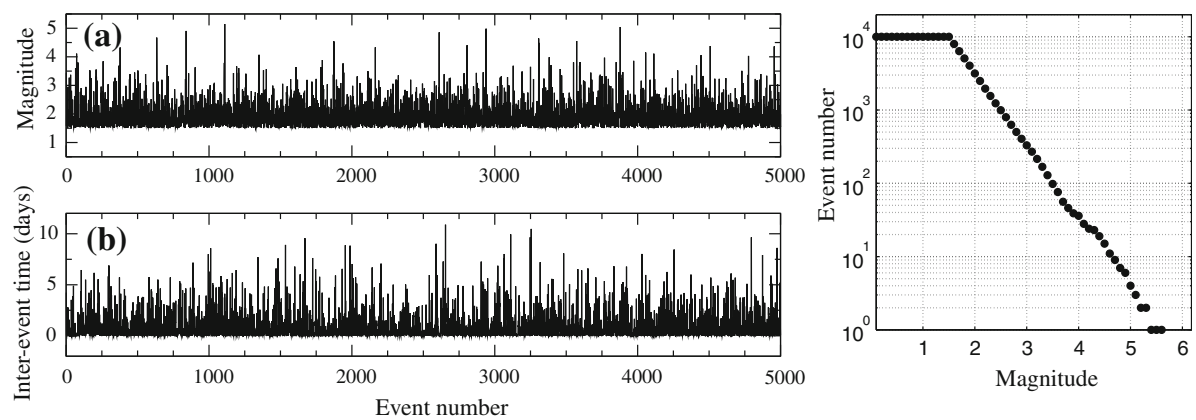

Fig. 1 : Synthetic earthquake series produced by the ETAS model (see text for parameter values). a MaLeftgnitude series. b Series of inter-event times. Right: Gutenberg-Richter curve of the synthetic magnitude series shown on the upper left 

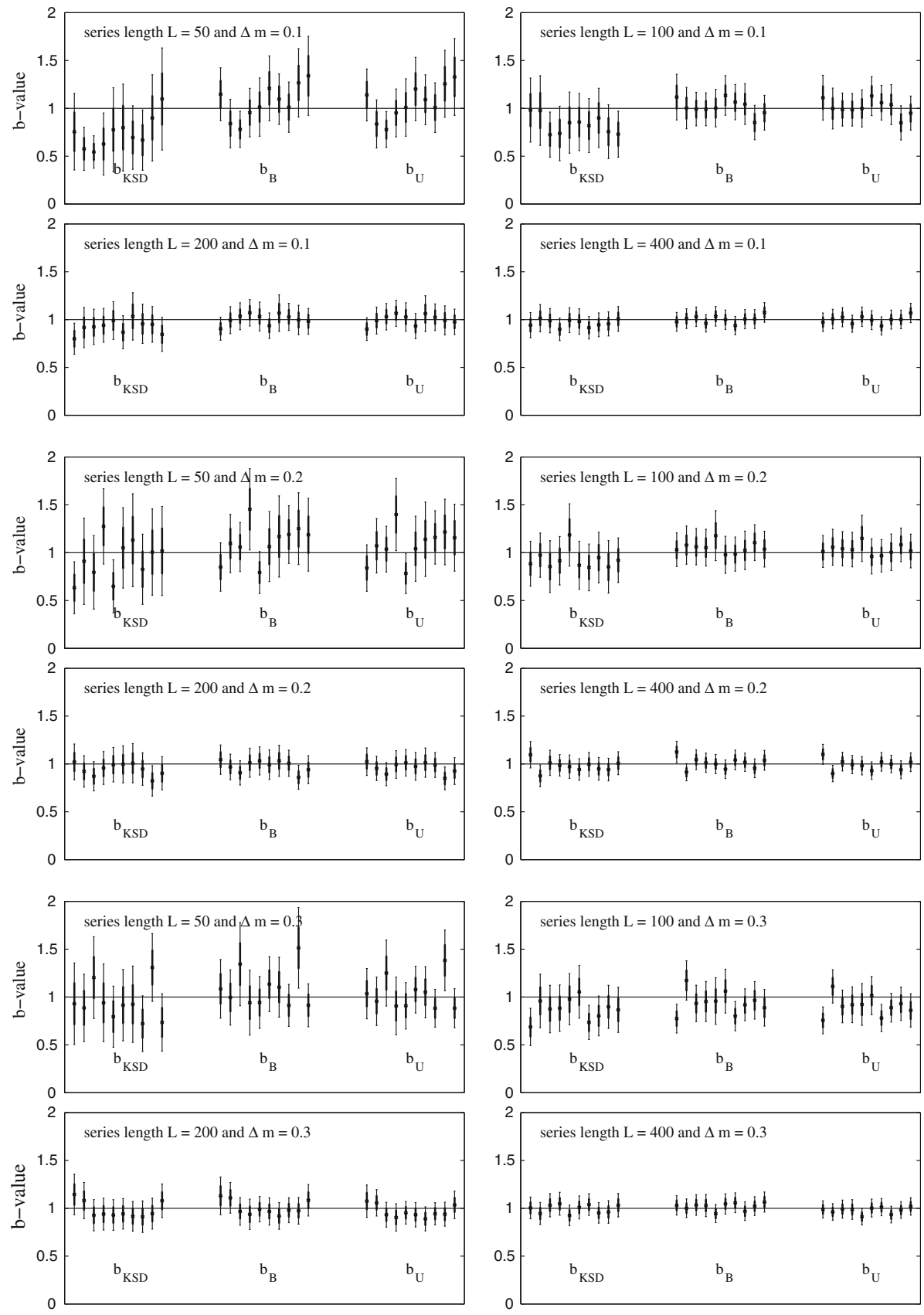

Fig. 2 Estimates for $b_{\mathrm{KSD}}, \quad b_{B}$, and $b_{U}$ obtained for 10 bootstrap runs (each with $2 \times 10^{5}$ replicated series) for $\Delta m=\{0.1,0.2,0.3\}$, and $L=\{50,100,200,400\}$. The thick and thin vertical bars are $1-\sigma$ and $2-\sigma$ error bars, respectively 

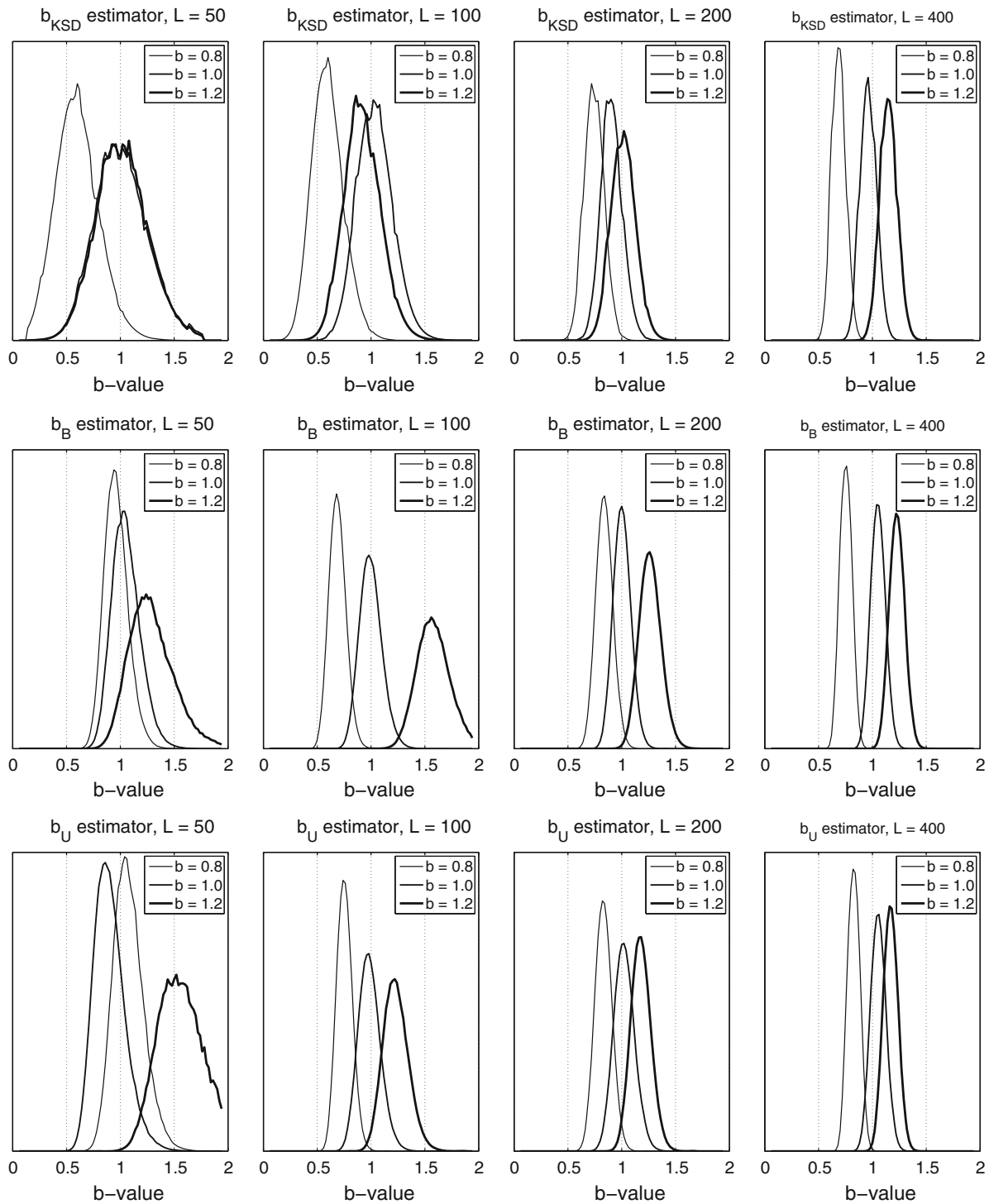

Fig. 3 Experimental probability density functions for $b_{\mathrm{KSD}}, b_{B}$, and $b_{U}$ obtained by bootstrapping a single ETAS series with $b=0.8$ (thin line), $b=1.0$ (medium line), and $b=1.2$ (thick line). The quantization level $\Delta m=0.1$

and $\Delta m$, the bias may be attributed to the particular realization of the series of magnitudes and not to a particular $b$ estimator.

The $b_{\mathrm{KSD}}$ estimates appear scattered in a wider range than for the $b_{B}$ and $b_{U}$ estimators. This results in a number of significantly biased estimates about three times larger for $b_{\mathrm{KSD}}$ than for the other two estimators. The abnormal high number of negatively biased estimates obtained for the $b_{\mathrm{KSD}}$ estimator at $L=50$ and $\Delta m=0.1$ is coherent with the results of the Monte Carlo runs which give $b_{\mathrm{KSD}}=0.78$ (Table 2). The $b_{U}$ and $b_{B}$ estimators perform equally well and slightly larger confidence intervals obtained with $b_{\mathrm{KSD}}$. These 
tests indicate that the random re-sampling of the bootstrap implemented in the present study correctly reproduces the statistical distribution of the $b$ estimator.

\subsection{Discrimination of different $b$-values from short series of magnitudes}

As a brief illustrative example of practical application, we apply the different bootstrap estimators discussed above to distinguish different $b$-values from experimental series with $\Delta m=0.1, L=\{50,100,200,400\}$, and $b=\{0.8,1.0,1.2\}$. As in the tests discussed in the preceding sections, a single series of magnitude is used for each value of $b$ and $L$, and the number of bootstrap series is $n=2 \times 10^{5}$. The experimental probability density functions (pdf) of the $b_{\mathrm{KSD}}, b_{B}$, and $b_{U}$ estimators are shown in Fig. 3. All estimators give poor results for $L=50$ with wide pdf making any $b$ discrimination unreliable. In one instance $\left(b_{\mathrm{KSD}}, \quad L=50\right)$, two pdf are almost indistinguishable, and in another one $\left(b_{U}\right.$, $L=50$ ), the $b=0.8$ and $b=1.0$ pdf appear inverted, giving a lower estimate of $b$ for the series generated with $b=1$. This is understandable in regard to the results in the top part of Fig. 2, showing that the bias occurring for $L=50$ may likely exceed the resolution necessary to distinguish among $b=\{0.8,1.0,1.2\}$. The situation appears better at $L=100$ for $b_{B}$ and $b_{U}$ but strong bias remains likely. All estimators give reliable results for $L=\{200,400\}$ with narrow distinguishable pdf.

\section{Conclusion}

The numerical tests performed in the present study provide some practical guidelines concerning the practical computation of the $b$-value with several common estimators. We considered the case of magnitude series with different lengths $L=50,100,200$, and 400, and different quantization levels $\Delta m=0.1,0.2$ and 0.3 to reach the limits of efficiency of the bootstrap procedure to obtain reliable estimates of the $b$-value. The results detailed in the present study allow to enumerate the following recommendations:

1. The Monte Carlo tests performed with series of exact magnitudes (Sect. 3.2 and Table 1) confirm that the $b_{L R}$ estimator has a negative bias of more than $5 \%$, while the $b_{\mathrm{MLE}}$ and $b_{\mathrm{KS}}$ estimators appear unbiased whatever the length $L$ of the series. The standard deviation of $b_{L R}$ is 2 or 3 times larger than the standard deviation of $b_{\mathrm{MLE}}$ which is well-predicted by formula (9). These results agree with the conclusions of Goldstein et al. (2004) and Newman (2005). We emphasize that the linear leastsquares $b_{L R}$ estimator must not be used to derive $b$-value estimates.

2. A Monte Carlo evaluation of the quantization of the magnitude values is done (Sect. $4.3)$ with $\Delta m=0.1,0.2$ and 0.3 for three discrete version of $b$ estimators $\left(b_{B}, b_{U}\right.$, and $\left.b_{\mathrm{KSD}}\right)$. The results summarized in Table 2 show that the $b_{B}$ estimator performs as well as the continuous $b_{\mathrm{MLE}}$ for all quantization levels and series lengths. Despite slightly altered performances, the $b_{U}$ estimator may also be considered reliable. Excepted for $L=400$ where it performs well, the $b_{\mathrm{KSD}}$ estimator is biased by about $10 \%$.

3. The bootstrap analysis performed in Sect. 5 and summarized in Table 2 show that bootstrapping retrieves the Monte Carlo statistics for $L=400$ and all quantization levels. For other values of $L$, all estimators display slightly degraded performances with bias of less than $10 \%$ except for the $b_{\mathrm{KSD}}$ estimator which may have a bias of more than $20 \%$. A large number $n=2 \times 10^{5}$ of bootstrapped series is necessary to obtain reliable statistical figures (Wehrens et al. 2000). 
4. By repeating bootstrap studies with different data series, we observed that, when present, a bias simultaneously affects all three $b$ estimators (Fig. 2). This allows us to conclude that the bias of the estimators is inherited from a sampling bias in the original data series to be bootstrapped. In such a case, bootstrap fails at correctly reproducing the statistical variability as obtained with the Monte Carlo method of Sect. 4.3 (unfortunately impossible to apply to real data). This bias problem is mainly observed for $L=50$ and 100 .

5. The analysis of short series of magnitude with $L \leq 100$ must be considered with much caution because of the significant probability of occurrence of strong biases. For such series, the analysis should be supplemented by a Monte Carlo analysis giving the probability of occurrence of a given value of the bias. This can be practically achieved by doing a statistical analysis of an ensemble of results like those shown on Fig. 2 for the relevant values of $L$ and $\Delta m$.

6. The last question raised in the present study addresses the resolution which can be achieved with the $b_{B}, \quad b_{U}$, and $b_{\mathrm{KSD}}$ estimators to distinguish series with different $b$-values. Our tests (Fig. 3) indicate that, despite small bias, all estimators are able to resolve $b$-value difference of $\Delta b \simeq 0.2$ for $L \geq 200$. In order to get reliable results, each time window must be bootstrapped and a Monte Carlo analysis of possible bias is recommended.

Acknowledgments We thank two anonymous Referees for their very constructive review that helped us to improve the manuscript. This is IPGP contribution 3320.

\section{Appendix: Derivation of unbiased MLEs}

In what follows, we assume that the magnitudes are quantized in intervals of width $\Delta m$, that is, the $L$ values of magnitudes forming the analyzed series belong to the finite and discrete set $\mathcal{S}=\left\{m_{n}=m_{\min }+n \Delta m n=0, \ldots, N\right\}$, where $m_{\text {min }}$ is the smallest quantized magnitude. Equation (4) implies that the probability density distribution of the magnitudes is a power law,

$$
p(m)=A 10^{-b m},
$$

where $A$ is a suitable normalizing constant. A bias in the MLE $b$-value estimate may appear if the average magnitude which appears in Eq. (8) is taken as the average of the quantized magnitudes of the analyzed series. This can be checked by considering the average magnitude, $\bar{m}_{n}$, computed over the $n$ 's bin interval $\left[m_{n-\frac{1}{2}}, m_{n+\frac{1}{2}}\right]$ :

$$
\begin{aligned}
\bar{m}_{n}=A \int_{m_{n-\frac{1}{2}}}^{m_{n+\frac{1}{2}}} m 10^{-b m} \mathrm{dm} \\
=\frac{m_{n-\frac{1}{2}}-m_{n+\frac{1}{2}} 10^{-b \Delta m}}{1-10^{-b \Delta m}}+\frac{1}{\ln (10) b} \\
=m_{n-\frac{1}{2}}+\frac{\Delta m}{1-10^{b \Delta m}}+\frac{1}{\ln (10) b} .
\end{aligned}
$$

In practice, the average magnitude associated with the $n$ 's bin is taken as $\overline{m_{b n}}=m_{n}=m_{\min }+n \Delta m$, that is, the center of the $\left[m_{n-\frac{1}{2}}, m_{n+\frac{1}{2}}\right]$ interval. The bias, $\delta$, is straightforwardly obtained from Eq. (14), 


$$
\begin{aligned}
\delta & =\overline{m_{b}}-\bar{m}_{n} \\
& =\frac{\Delta m}{2} \times \frac{1+10^{-b \Delta m}}{1-10^{-b \Delta m}}-\frac{1}{\ln (10) b} .
\end{aligned}
$$

Observe that the bias is constant whatever the interval considered.

The average of the discretized data is given by,

$$
\begin{aligned}
{\overline{m_{b N}}} & =\sum_{n=1}^{N} P_{n} \overline{m_{b}} \\
& =\sum_{n=1}^{N} P_{n} \bar{m}_{n}+\delta
\end{aligned}
$$

with $\sum_{n=1}^{N} P_{n}=1$ where the $P_{n}$ 's are the probabilities integrated over the bins,

$$
\begin{aligned}
P_{n} & =\frac{-\ln (10) b}{10^{-b m_{N+\frac{1}{2}}}-10^{-b m_{-\frac{1}{2}}}} \int_{m_{n-\frac{1}{2}}}^{m_{n+\frac{1}{2}}} 10^{-b m} \mathrm{dm} \\
& =\frac{1}{10^{-b m_{N+\frac{1}{2}}}-10^{-b m_{-\frac{1}{2}}}\left[10^{-b m}\right]_{m_{n-\frac{1}{2}}^{m_{n+\frac{1}{2}}}}^{-b m_{n-\frac{1}{2}}}} \\
& =\frac{10^{-b m_{n+\frac{1}{2}}}-10^{-b m_{N+\frac{1}{2}}}-10^{-b m_{-\frac{1}{2}}}}{10^{-b \Delta m}} \\
& =10^{-b\left(n-\frac{1}{2}\right) \Delta m} \frac{1-10^{-b \Delta m}}{1-10^{-b N \Delta m}} .
\end{aligned}
$$

Considering Eq. (16) again, we obtain,

$$
\bar{m}_{b N}=\bar{m}_{N}+\frac{\Delta m}{2} \times \frac{1+10^{-b \Delta m}}{1-10^{-b \Delta m}}-\frac{1}{\ln (10) b} .
$$

The computation of the continuous average, $\bar{m}_{N}$, involves the same kind of integral as in Eq. (14) but for the whole interval $\left[m_{-\frac{1}{2}}, m_{N+\frac{1}{2}}\right]$ spanning the range of the quantified magnitudes. This results in

$$
\bar{m}_{N}=m_{-\frac{1}{2}}+\frac{N \Delta m}{1-10^{b N \Delta m}}+\frac{1}{\ln (10) b}
$$

and, inserting this last expression in Eq. (18), we obtain,

$$
\begin{aligned}
\bar{m}_{b N} & =m_{-\frac{1}{2}}+\frac{\Delta m}{2}+\frac{\Delta m 10^{-b \Delta m}}{1-10^{-b \Delta m}}-\frac{\Delta m N 10^{-b N \Delta m}}{1-10^{-b N \Delta m}} \\
& =m_{\min }+\frac{\Delta m 10^{-b \Delta m}}{1-10^{-b \Delta m}}-\frac{\Delta m N 10^{-b N \Delta m}}{1-10^{-b N \Delta m}} .
\end{aligned}
$$

In the expression above, the quantity $\bar{m}_{b N}$ is the average magnitude which is effectively computed from the data. So, Eq. (20) may be used to derive a value for $b$ (Bender 1983).

Various degrees of approximation may be applied to the general expression (20). The first one consists in considering that $m_{N+\frac{1}{2}} \rightarrow \infty$ (or, equivalently, that $N \rightarrow \infty$ ) so that the rightmost term of the second member of Eq. (20) vanishes. This approximation is almost 
always satisfied since generally $N \geq 10$. The average of the discrete magnitudes then reads,

$$
\overline{m_{b}}=m_{\min }+\Delta m \frac{10^{-b \Delta m}}{1-10^{-b \Delta m}} .
$$

The $b$-value estimate obtained by solving Eq. (21) is given by,

$$
b_{B}=\frac{1}{\ln (10) \Delta m} \ln \left(1+\frac{\Delta m}{\overline{m_{b} \infty}-m_{\min }}\right),
$$

identical to the one used by Tinti and Mulargia (1987).

A second simplification occurs if $\Delta m \rightarrow 0$, which gives,

$$
\overline{m_{b} \infty}=m_{-\frac{1}{2}}+\frac{1}{\ln (10) b},
$$

where the asymptotic value for the last term was obtained by applying l'Hospital rule. Solving this equation leads to the unbiased $b$-value estimate given by Utsu (1965),

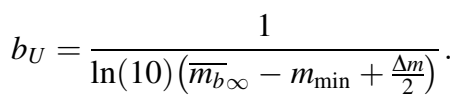

\section{References}

Abadie A, Imbens GW (2008) On the failure of the bootstrap for matching estimators. Econometrica 76:1537-1557

Aki K (1965) Maximum likelihood estimate of $b$ in the formula $\log (N)=a-b M$ and its confidence limits. Bull Earthq Res Inst Tokyo Univ 43:237-239

Amitrano D (2003) Brittle-ductile transition and associated seismicity: experimental and numerical studies and relationship with the b value. J Geophys Res 108(B1):2044. doi:10.1029/2001JB000680

Ayele A, Kulhánek O (1997) Spatial and temporal variation of seismicity in the horn of Africa from 1960 to 1993. Geophys J Int 130:805-810

Bender B (1983) Maximum likelihood estimation of $b$ values for magnitude grouped data. Bull Seismol Soc Am 73:831-851

Bouhadad Y, Laouami N (2002) Earthquake hazard assessment in the oran region (Northwest Algeria). Nat Hazards 26:227-243

Castellaro S, Mulargia F, Kagan YY (2006) Regression problems for magnitudes. Geophys J Int 165:913-930. doi:10.1111/j.1365-246X.2006.02955.x

Davison AC, Hinkley DV (1997) Bootstrap methods and their application. Cambridge University Press, Cambridge, p 592

Efron B (1992) Six questions raised by the bootstrap. In: LePage R, Billard L (eds) Exploring the limits of bootstrap. Wiley, New York

Efron B (1982) The jacknife, the bootstrap, and other resampling plans, CBMS monograph \#38. Society for Industrial and Applied Mathematics, Philadelphia

Enescu B, Kiyoshi I, Struzik ZR (2006) Wavelet-based multiscale resolution analysis of real and simulated time-series of earthquakes. Geophys J Int 163:63-74. doi:10.1111/j.1365-246X.2005.02810.x

Felzer KR, Becker TW, Abercrombie RE, Ekström G, Rice JR (2002) Triggering of the $1999 M_{W} 7.1$ Hector mine earthquake by aftershocks of the $1992 M_{W} 7.3$ landers earthquake. J Geophys Res 107(B9):2190. doi:10.1029/2001JB000911

Frohlich C (2004) To $b$ or not to $b$ ? Is there value in $b$ value research ? AGU fall meeting, S22A. http://www.agu.org/meetings/ fm04

Gerstenberger M, Wiemer S, Gardini D (2001) A systematic test of the hypothesis that the $b$-value varies with depth in California. Geophys Res Lett 28:57-60

Goldstein ML, Morris SA, Yen GG (2004) Problems with fitting to the power-law distribution. Eur Phys J B $41: 255-258$ 
Gutenberg R, Richter CF (1944) Frequency of earthquakes in California. Bull Seism Soc Am 34:185-188 Kagan Y (1999) The universality of the frequency-magnitude relationship. Pure Appl Geophys 155:537-574

Main I (2000) Apparent breaks in scaling in the earthquake cumulative frequency-magnitude distribution: fact or artifact? Bull Seismol Soc Am 90:86-97

Marzocchi W, Sandri L (2003) A review and new insights on the estimation of the $b$-value and its uncertainty. Ann Geophys 46:1271-1282

McNutt SR (2005) Volcanic seismology. Annu Rev Earth Planet Sci 32:461-491

Meyer U, Sobiesiak M, Schmidt S, Goetze H, Krawczyk C (2004) Identifying asperities: correlating $b$-value and isostatic residual anomaly maps. AGU fall meeting, S13A. http://www.agu.org/meetings/fm04

Murru M, Console R, Lisi A, Montuori C (2004) Mapping of the $b$-value anomalies in Colfiorito tectonic zone (Central Italy) and beneath Mt. Etna volcano (Sicily, Italy). AGU fall meeting, S13A. http:// www.agu.org/meetings/fm04

Newman MEJ (2005) Power laws, Pareto distributions and Zipfs law. Contemp Phys 43:323-351

Nuannin P, Kulhánek O, Persson L (2005) Spatial and temporal $b$-value anomalies preceding the devastating off coast of NW Sumatra earthquake of December 26, 2004. Geophys Res Lett 32:L11307. doi: 10.1029/2005GL022679

Ogata Y (1985) Statistical models for earthquake occurrences and residual analysis for point processes, research memo. (technical report) 288. Institute of Statistical Mathematics, Tokyo

Ogata Y (1988) Statistical models for earthquake occurrences and residual analysis for point processes. J Am Stat As 83:9-27

Okal E, Kirby SH (1995) Frequency-moment distribution of deep earthquake: implication for the seismogenic zone at the bottom of slabs. Phys Earth Planet Inter 92:169-187

Papadopoulos GA, Latoussakis I, Daskalaki E, Diakogianni G, Fokaefs A, Kolligri M, Liadopoulou K, Orfanogiannaki K, Pirentis A (2006) The East Aegean Sea strong earthquake sequence of October November 2005: lessons learned for earthquake prediction from foreshocks. Nat Hazards Earth Syst Sci 6:895-901

Papadopoulos GA, Baskoutas I (2009) New tool for the spatio-temporal variation analysis of seismic parameters. Nat Hazards Earth Syst Sci 9:859-864

Peléez Montilla JA, Hamdache M (2004) Comment on the paper Earthquake Hazard Assessment in the Oran Region (Northwest Algeria) by Y. Bouhadad and N. Laouami. Nat Hazards 32:155-159

Scholz CH (1968) The frequency-magnitude relation of microfracturing in rock and its relation to earthquakes. Bull Seismol Soc Am 58:399-415

Schorlemmer D, Zechar D, Wermer MJ, Field EH, Jackson DD, Jordan TH, the RELM Working Group (2010) First results of the regional earthquake likelihood models experiment. Pure Appl Geophys 167:859-876

Schorlemmer D, Wiemer S, Wyss M (2005) Variations in earthquake-size distribution across different stress regimes. Nature 437(22):539-542

Schorlemmer D, Neri G, Wiemer S, Mostaccio A (2003) Stability and significance tests for b-value anomalies: example from the Tyrrhenian Sea. Geophys Res Lett 30(16):1835. doi:10.1029/2003 GL017335

Sornette D, Werner MJ (2005) Constraints on the size of the smallest triggering earthquake from the ETAS model, Baths law, and observed aftershock series. J Geophys Res 110(B08):304. doi:10.1029/2004 JB003535

Telesca L, Rouai M, Cherkaoui TE (2009) Time-clustering behavior in the sequence of the aftershocks of the Al-Hoceima (Morocco) 24 February 2004 earthquake. Nat Hazards Earth Syst Sci 9:2063-2066

Tinti S, Mulargia F (1987) Confidence intervals of b-values for grouped magnitudes. Bull Seismol Soc Am $77: 2125-2134$

Triep EG, Sykes LR (1997) Frequency of occurrence of moderate to great earthquakes in intracontinental regions: implications for change in stress, earthquake prediction, and hazard assessment. J Geophys Res 102:9923-9948

Utsu T (1961) Statistical study on the occurence of aftershocks. Geophys Mag 30:521-605

Utsu $\mathrm{T}$ (1965) A method for determining the value of $b$ in a formula $\log n=a-b M$ showing the magnitude-frequency relation for earthquakes. Geophys Bull Hokkaido Univ 13:99-103

Wehrens R, Putter H, Buydens LMC (2000) The bootstrap: a tutorial. Chemom Intell Lab Syst 54:35-52

Wiemer S, Wyss M (2000) Minimum magnitude of completeness in earthquake catalogs: examples from Alaska, the Western United States and Japan. Bull Seismol Soc Am 90:859-869

Wiemer S, Benoit J (1996) Mapping the $b$-value anomaly at $100 \mathrm{~km}$ depth in the Alaska and New Zealand subduction zones. Geophys Res Lett 23:1557-1560

Wiemer S, McNutt S (1997) Variations in frequency-magnitude distribution with depth in two volcanic areas: Mount St. Helens, Washington, and Mt. Spurr, Alaska. Geophys Res Lett 24:189-192 
Wiemer S, McNutt SR, Wyss M (1998) Temporal and three-dimensional spatial analyses of the frequencymagnitude distribution near Long Valley Caldera, California. Geophys J Int 134:409-421

Wyss M (1973) Towards a physical understanding of the earthquake frequency distribution. Geophys J R Astr Soc 31:341-359

Wyss M, Habermann RE (1988) Precursory quiescence before the August 1982 Stone Canyon, San Andrea Fault, earthquakes. Pure Appl Geophys 126:333-356

Wyss M, Shimazaki K, Wiemer S (1997) Mapping active magma chambers by b values beneath the Off-Ito volcano, Japan. J Geophys Res 102:20413-20422

Wyss M, Klein F, Nagamine K, Wiemer S (2001) Anomalously high $b$-values in the South Flank of Kilauea volcano, Hawaii: evidence for the distribution of magma below Kilauea's East rift zone. J Volcanol Geotherm Res 106:23-37

Young A (1994) Bootstrap: more than a stab in the dark? Stat Sci 9:382-395

Zhao YZ, Wu ZL (2008) Mapping the $b$-values along the Longmenshan fault zone before and after the 12 May 2008, Wenchuan, China, $M_{S}=8.0$ earthquake. Nat Hazards Earth Syst Sci 8:1375-1385

Zöller G, Hainzl S, Kurths J, Zschau J (2002) A systematic test on precursory seismic quiescence in Armenia. Nat Hazards 26:245-263 